\title{
MAPPING AND MASS MEASUREMENT OF THE COLD DUST IN NGC 205 WITH SPITZER
}

\author{
F. R. Marleau, ${ }^{1}$ A. Noriega-Crespo, ${ }^{1}$ K. A. Misselt $^{2}{ }^{2}$ K. D. Gordon, ${ }^{2}$ C. W. Engelbracht,${ }^{2}$ G. H. Rieke, ${ }^{2}$ \\ P. Barmby, ${ }^{3}$ S. P. Willner, ${ }^{3}$ J. Mould, ${ }^{4}$ R. D. Gehrz, ${ }^{5}$ and C. E. Woodward ${ }^{5}$ \\ Received 2006 January 27; accepted 2006 April 8
}

\begin{abstract}
We present observations at 3.6, 4.5, 5.8, 8, 24, 70, and $160 \mu \mathrm{m}$ of NGC 205, the dwarf elliptical companion of M31, obtained with the Spitzer Space Telescope. The point-source-subtracted images at 8 and $24 \mu \mathrm{m}$ display a complex and fragmented infrared emission coming from both very small dust particles and larger grains. The extended dust emission is spatially concentrated in three main emission regions, seen at all wavelengths from 8 to $160 \mu \mathrm{m}$. These regions lie approximately along NGC 205's semimajor axis and range from $\sim 100$ to $300 \mathrm{pc}$ in size. On the basis of our mid-/far-infrared flux density measurements alone, we derive a total dust mass estimate on the order of $3.2 \times 10^{4} M_{\odot}$, mainly at a temperature of $\sim 20 \mathrm{~K}$. The gas mass associated with this component matches the predicted mass returned by the dying stars from the last burst of star formation in NGC 205 ( $\sim 0.5 \mathrm{Gyr}$ ago). Analysis of the Spitzer data combined with previous $1.1 \mathrm{~mm}$ observations over a small central or "Core" region (18" diameter) suggests the presence of very cold ( $T \sim 12 \mathrm{~K}$ ) dust and a dust mass about 16 times higher than is estimated from the Spitzer measurements alone. Assuming a gas-to-dust mass ratio of 100 , these two data sets, i.e., with and without the millimeter observations, suggest a total gas mass in the range from $3.2 \times 10^{6}$ to $5 \times 10^{7} M_{\odot}$.
\end{abstract}

Subject headings: dust, extinction — galaxies: dwarf — galaxies: individual (NGC 205) — galaxies: ISM — infrared: galaxies — infrared: ISM — ISM: structure

\section{INTRODUCTION}

The galaxies of the Local Group, because of their proximity and our ability to carry out high spatial resolution studies in nearby systems, provide us with powerful templates with which to understand the chemical, morphological, and kinematical characteristics, plus the star formation history, of more distant galactic systems. The local dwarf galaxies (LDGs) in particular have been subject to numerous recent studies of their stellar populations and star formation (for summaries, see, for example, Hodge 1989, Mateo 1998, and Grebel 2005). Dwarf elliptical galaxies follow a different "fundamental plane" than true elliptical galaxies (Ferguson \& Binggeli 1994); nevertheless, they do share many common characteristics, in particular the presence of a mostly old stellar population (Mateo 1998). One of these local dwarf systems is NGC 205, a low surface brightness dwarf elliptical companion of M31. NGC 205 is interesting because of its very conspicuous dark clouds, which were detected in some of the earliest photographic plates of M31 and its surroundings (Baade 1944; Hodge 1973; NGC 205 lies $\sim 36^{\prime}$ northwest of the M31 nucleus). These visually dark clouds reveal the presence of an interstellar medium in a stellar population dominated by old stars; that is, in an elliptical galaxy.

There are several indicators for an intermediate and young stellar population, as well as for gas enrichment, in NGC 205. A handful of blue and UV-bright stars have been detected in its nucleus (Baade 1951; Hodge 1973; Bertola et al. 1995), with ages

\footnotetext{
1 Spitzer Science Center, Mail Code 220-6, California Institute of Technology, 1200 East California Boulevard, Pasadena, CA 91125.

2 Steward Observatory, University of Arizona, 933 North Cherry Avenue, Tucson, AZ 85721.

${ }^{3}$ Harvard-Smithsonian Center for Astrophysics, 60 Garden Street, Cambridge, MA 02138.

4 National Optical Astronomy Observatory, P.O. Box 26732, Tucson, AZ 85726.

5 Department of Astronomy, University of Minnesota, 116 Church Street SE, Minneapolis, MN 55455.
}

ranging from $\sim 5 \times 10^{8}$ to a few times $10^{6} \mathrm{yr}$. In terms of metallicity, the location of its giant branch implies a mean value of $[\mathrm{Fe} / \mathrm{H}]=-0.85 \pm 0.2$ (Mould et al. 1984), higher than for most LDGs, which have values ranging from -1.1 to -2.2 (Mateo 1998). Moreover, a stellar population study of the resolved bright near-IR stars in NGC 205 (Davidge 2003) indicates that the brightest asymptotic giant branch (AGB) stars formed a few tenths of a Gyr ago. All these indicators suggest an episode of star formation in NGC 205 that took place a few times $10^{8}$ yr ago and a recent replenishment of the interstellar material by this population of evolved stars. Although there is no consensus on the process that triggered such a burst, gravitational tidal interaction of NGC 205 with M31 has always been an appealing mechanism because it simultaneously explains the twisted surface brightness isophotes (Hodge 1973; Choi et al. 2002), the tidal trail of blue metal-poor red giant branch stars (McConnachie et al. 2004; Ibata et al. 2001), and the kinematically distinct behavior and morphology of its interstellar medium (ISM), particularly of the $\mathrm{H}$ I gas (Young \& Lo 1997).

The ISM in NGC 205 has some peculiarities. On the basis of the last burst of star formation $\sim 5 \times 10^{8} \mathrm{yr}$ ago (Wilcots et al. 1990; Bertola et al. 1995), one can estimate the amount of gas injected back into the ISM, which is $\sim 2.6 \times 10^{6} M_{\odot}$ (following Faber \& Gallagher 1976). This mass is about 3 times larger than that measured by Sage et al. (1998), $7.2 \times 10^{5} M_{\odot}\left(\mathrm{H}_{2}\right.$ [from CO] plus $\mathrm{H}$ I, scaled by a factor of 1.4 to include helium). This difference in masses led to the idea that gas is being removed by the young stars in NGC 205, either by stellar winds or by supernova explosions (Welch et al. 1998). Recently Haas (1998) carried out photometric observations between 120 and $200 \mu \mathrm{m}$ with the $I n$ frared Space Observatory (ISO) that, combined with a millimetric measurement of the central 18" (Fich \& Hodge 1991), yielded large amounts of cold dust with mass $0.2-1.2 \times 10^{4} M_{\odot}$ at $T \sim$ $10 \mathrm{~K}$. If we assume a gas-to-dust mass ratio of 100 , the cold dust traces nearly $10^{6} M_{\odot}$ of gas at the center. There is vast observational evidence for "warm" and "cool" dust in elliptical galaxies 


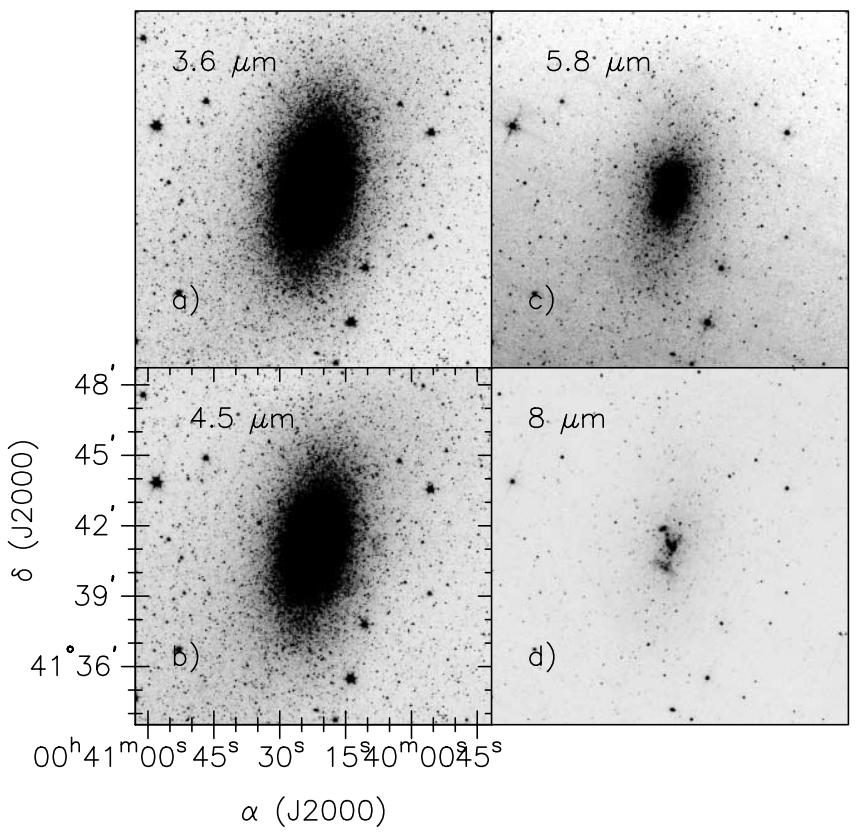

FIG. 1.-IRAC 3.6, 4.5, 5.8, and $8 \mu \mathrm{m}$ images of NGC 205. The FOV of each image is $15^{\prime} \times 15^{\prime}$, with north up and east to the left.

based on mid-/far-infrared data (see, e.g., Jura et al. 1987; Knapp et al. 1989; Ferrari et al. 2002; Temi et al. 2004; Xilouris et al. 2004). The initial Infrared Astronomical Satellite (IRAS) measurements were limited to the detection of temperatures of $\sim 30 \mathrm{~K}$ traceable by the $100 \mu \mathrm{m}$ fluxes. This led to the possibility of missing a colder component with nearly $90 \%$ of the dust mass at lower temperatures $(T \sim 15-20 \mathrm{~K})$ that was radiating at longer wavelengths ( $>100 \mu \mathrm{m}$; see, e.g., Devereux \& Young 1990). The longer wavelength far-infrared observations $(>100 \mu \mathrm{m})$ combined with millimeter and submillimeter measurements have confirmed this picture in several early-type galaxies, where the bulk of the dust mass is cold $(\sim 20 \mathrm{~K})$ and nearly an order of magnitude larger than previously estimated (Wiklind \& Henkel 1995; Temi et al. 2004).

This study presents new mid-/far-infrared images of NGC 205 obtained with the Spitzer Space Telescope (Spitzer). The higher sensitivity and spatial resolution enable us for the first time to look directly into the detailed structure and the properties of the dust clouds in NGC 205 and to evaluate their dust properties and content. The outline of the paper is as follows. In $\S 2$, we describe the Spitzer observations and the data reduction. The Spitzer images are presented in $\S 3$, along with a description of the mid-/far-IR emission morphology and a comparison with other tracers of the ISM. Additional image processing is discussed in $\S 4$. Dust mass measurements and gas mass estimates are derived in $\S 5$, using two different methods. In $\S 6$, we discuss the results, including some of the previous mass estimates and theoretical expectations. We conclude with a summary of our findings in $\S 7$.

\section{OBSERVATIONS}

The mid-IR observations were obtained with the Infrared Array Camera (IRAC), the infrared camera on board Spitzer (Fazio et al. 2004) on 2005 January 20 as part of the GO1 program to map M31 (PI: P. B.; program ID 3126). The region around NGC 205 was observed with four dithered 30 s frames per sky position; the IRAC field of view (FOV) is $5^{\prime}$. The images obtained with channels 1 and 2 (at 3.6 and $4.5 \mu \mathrm{m}$ ) are used mostly to determine the stellar contribution to the overall spectral energy distribution

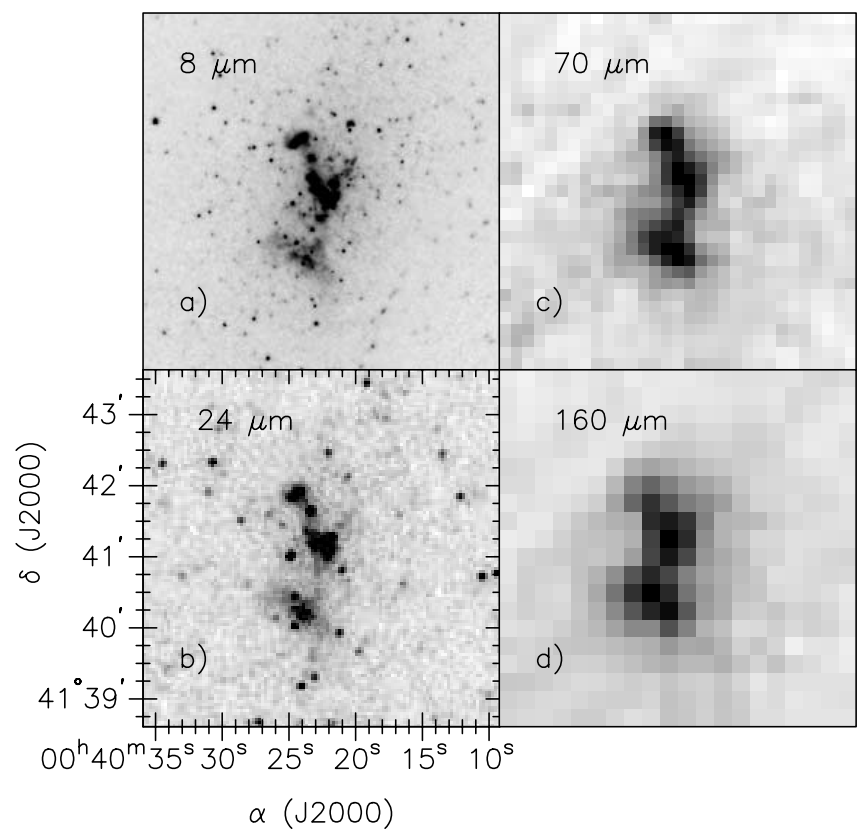

FIG. 2.-IRAC $8 \mu \mathrm{m}$ and MIPS 24, 70, and $160 \mu \mathrm{m}$ images of NGC 205. The FOV of each image is $5^{\prime} \times 5^{\prime}$, with north up and east to the left.

of NGC 205. Channels 3 and 4 (at 5.8 and $8 \mu \mathrm{m}$ ) contain the most information about the dust properties; channel 4 in particular, with a passband centered at $7.9 \mu \mathrm{m}$, includes some of the strongest polycyclic aromatic hydrocarbon (PAH) emission features known (e.g., bands at 7.7 and $8.6 \mu \mathrm{m}$ ); these are considered some of the best tracers of very small particles (see, e.g., Li \& Draine 2001; Draine \& Li 2001 and references therein). The basic calibrated data produced by the Spitzer Science Center (SSC) pipeline (version 11) were combined with the SSC MOPEX software to produce final mosaics with pixel size 1".2 (all channels) and spatial resolutions of less than $2^{\prime \prime}$ FWHM.

The far-IR data were obtained with the multiwavelength photometer MIPS (the Multiband Imaging Photometer for Spitzer) on board Spitzer on 2004 August 25 (program ID 99). These observations were taken as part of the large M31 mosaic consisting of seven scan legs, between 0.75 and $1^{\circ} .25$ long, with a $148^{\prime \prime}$ overlap between scan legs. The scanning was done at the medium scan rate, covering a total area of $1^{\circ} \times 3^{\circ}$ approximately oriented along the major axis of M31, with a total observing time of $17.1 \mathrm{hr}$. The NGC 205 observations themselves have a depth of 84 s per pixel. The details of the observations and reductions are given by Gordon et al. (2006). In the three MIPS bands a background subtraction has been performed, which removes some timedependent artifacts along the scan legs. In the 70 and $160 \mu \mathrm{m}$ images, some stripping remains due to a nonlinear time response of the Ge:Ga arrays; however, their overall effect on the flux density around NGC 205 is very small $(\sim 1 \%-2 \%)$, and this is included in our uncertainties (see $\S 4.1$ ). The final 24, 70, and $160 \mu \mathrm{m}$ images have spatial resolutions of $6^{\prime \prime}, 18^{\prime \prime}$, and $40^{\prime \prime}$ FWHM, respectively (Rieke et al. 2004). The mosaic pixel scales are 2".49 at $24 \mu \mathrm{m}, 9$ 9.85 at $70 \mu \mathrm{m}$, and $16^{\prime \prime} 0$ at $160 \mu \mathrm{m}$.

The Spitzer data are complemented with ISOCAM observations of NGC 205 from the $I S O$ archive. The archive data (Off Line Processing, version 10.0) was rereduced using the CIA (Cam Interactive Analysis, version 4.0) software to improve mainly on the deglitching, following the rest of the standard reduction steps (Coulais \& Abergel 2000; Blommaert et al. 2003). The data set includes images at $14.3 \mu \mathrm{m}$ covering a $204^{\prime \prime} \times 216^{\prime \prime}$ field of view 


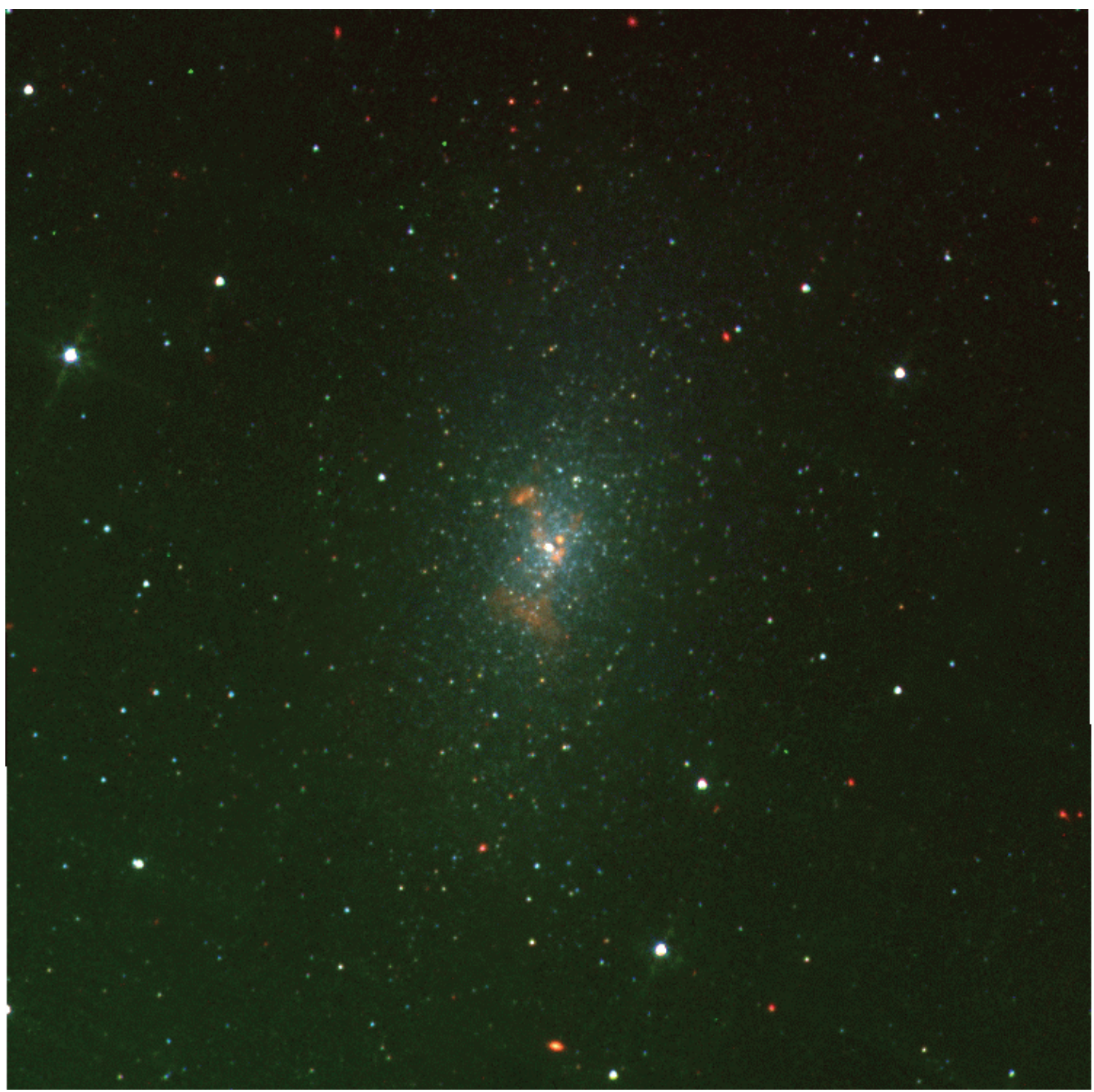

FIG. 3.-Three-color image of NGC 205 as seen by IRAC at 3.6 (blue), 5.8 (green), and $8 \mu \mathrm{m}$ (red). The image emphasizes the dust cloud distribution seen at longer wavelengths over a $15^{\prime} \times 15^{\prime}$ region, with north up and east to the left.

and with $6^{\prime \prime}$ per pixel. All the measurements were performed with background subtraction and were compared with the global estimates of Xilouris et al. (2004) for consistency.

For both IRAC and MIPS we used a $15^{\prime} \times 15^{\prime}$ section of the M31 maps centered on the position of NGC $205[\alpha(\mathrm{J} 2000.0)=$ $\left.00^{\mathrm{h}} 40^{\mathrm{m}} 22^{\mathrm{s}} \cdot 1, \delta(\mathrm{J} 2000.0)=41^{\circ} 41^{\prime} 07^{\prime \prime} \cdot 1\right]$. This region is large enough to encompass most of the visual size of the galaxy, $9^{\prime} \times$ $19^{\prime}$ (25 B mag $\operatorname{arcsec}^{-2}$; Nilson 1973), and certainly all the mid-/ far-IR emission (Rice et al. 1988). We adopt a distance to NGC 205 of $815 \pm 35 \mathrm{kpc}$ (Mateo 1998; McConnachie et al. 2004) throughout this work; at that distance, spatial structures $1^{\prime \prime}$ across measure $\sim 3.9 \mathrm{pc}$. This tentatively puts NGC 205 behind M31, which is at a distance of $780 \mathrm{kpc}$ (Rich et al. 2005; McConnachie et al. 2005).

\section{MORPHOLOGY OF INFRARED EMISSION}

Figures 1 and 2 show the images of NGC 205 taken with IRAC at 3.6 and $4.5 \mu \mathrm{m}$ (stellar emission dominant; see Table 4) and at $5.8,8,24,70$, and $160 \mu \mathrm{m}$ (dust emission dominant; see Table 4). Figure 3 displays a three-color image of NGC 205, where the dust clouds at 5.8 and $8 \mu \mathrm{m}$ stand out within the stellar background detected by the shorter wavelength bands. The higher angular resolution images at 8 and $24 \mu \mathrm{m}$ display a spatially complex and fragmented extended emission. We expect that the main contribution to the $8 \mu \mathrm{m}$ emission arises from aromatic molecules and, at $24 \mu \mathrm{m}$, from submicron-sized grains ( Li \& Draine 2002; Xilouris et al. 2004). The overlay of these two wavelengths (Fig. 4) shows the close spatial relationship between these two dust distributions. However, the $8 \mu \mathrm{m}$ image shows more extended emission than that at $24 \mu \mathrm{m}$ (see Fig. 5).

The morphology of the diffuse emission is complex and clumpy; nevertheless, we can clearly identify three spatially distinct emission regions: the largest region, to the south, with a size of $\sim 76^{\prime \prime} \times 37^{\prime \prime}(\sim 300 \mathrm{pc} \times 146 \mathrm{pc})$, the central region, with a size of $\sim 49^{\prime \prime} \times 28^{\prime \prime}(\sim 193 \mathrm{pc} \times 110 \mathrm{pc})$, and the smallest region, to the north, with a size of $\sim 33^{\prime \prime} \times 31^{\prime \prime}(\sim 130 \mathrm{pc} \times$ $123 \mathrm{pc}$ ) (see Fig. 4 and Table 1). Even at the lower resolution of the 70 and $160 \mu \mathrm{m}$ bands (see Fig. 2), where the bulk of the emission is produced by dust emission from the larger grains ( 0.1-0.5 $\mu$ m; Désert et al. 1990; Li \& Draine 2001), one can easily identify the same three large regions seen at shorter wavelengths. We have included also a "Core" region with a diameter of 18."4 for which $1.1 \mathrm{~mm}$ observations are available (Fich \& Hodge 1991). 


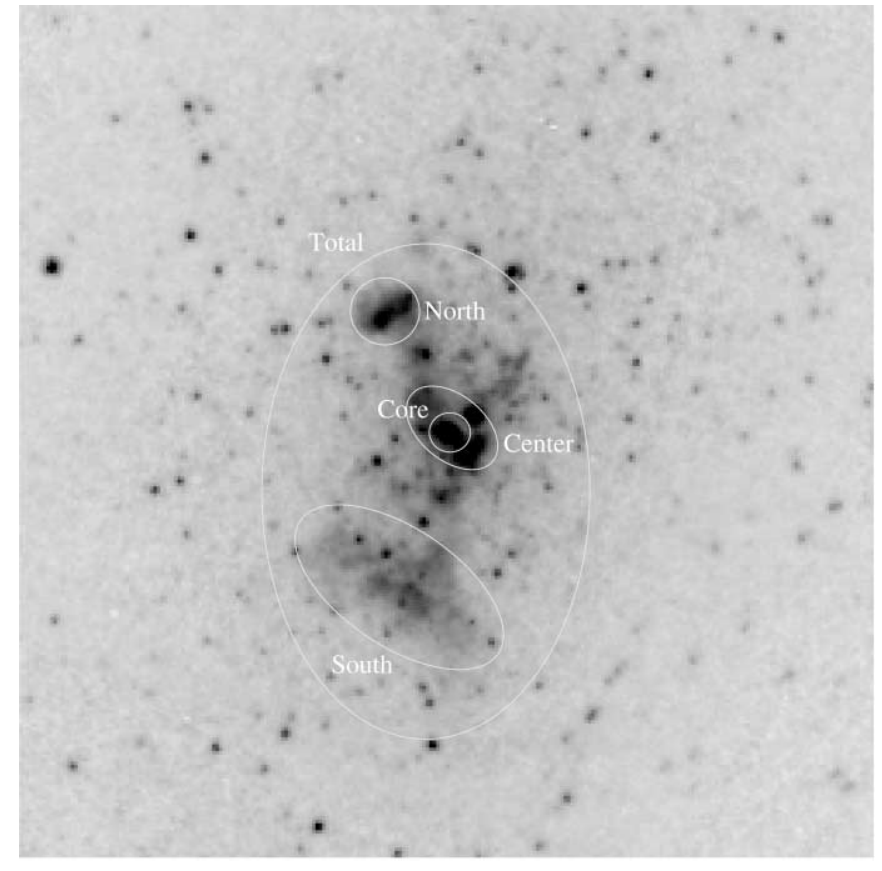

FIG. 4.- Three main emission regions, "North," "Center," and "South," as well as the "Core" and "Total" regions (as defined in Table 1), overlaid on the IRAC $8 \mu \mathrm{m}$ image of NGC 205 . The FOV is $5^{\prime} \times 5^{\prime}$, with north up and east to the left.

The $14.3 \mu \mathrm{m}$ ISOCAM observations, however, cover a smaller FOV, and the final mosaic has some vignetting that leaves an effective FOV of $\sim 192^{\prime \prime}$ radius. Although our mosaic is a bit larger than that presented by Xilouris et al. (2004; see their Fig. A.1), the "South" and "Total" regions are 3\% and 6\% smaller at $14.3 \mu \mathrm{m}$, respectively, than at all other wavelengths (see Table 3). Therefore, the emission not included directly in these two regions, given the very low surface brightness at $14.3 \mu \mathrm{m}$, is small.

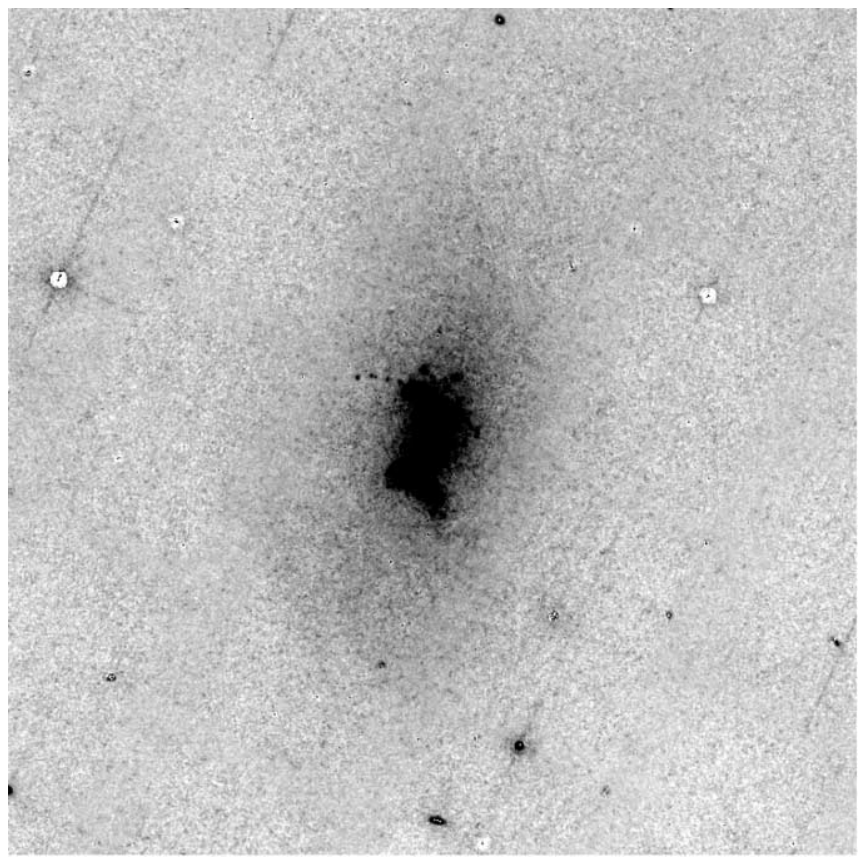

FIG. 5.-IRAC $8 \mu \mathrm{m}$ gray-scale image of NGC 205, where point sources have been removed to reveal more clearly the extended diffuse emission. The image is displayed at high contrast to enhance the faint emission surrounding the bright central region. The FOV is $15^{\prime} \times 15^{\prime}$, with north up and east to the left.
TABLE 1

NGC 205 EMISSION REGIONS

\begin{tabular}{|c|c|c|c|}
\hline Region $^{\mathrm{a}}$ & $\alpha(\mathrm{J} 2000.0)$ & $\delta(\mathrm{J} 2000.0)$ & $\begin{array}{c}\text { Area } \\
\left(\operatorname{arcmin}^{2}\right)\end{array}$ \\
\hline 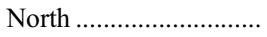 & 004024.00 & 414149.9 & 0.24 \\
\hline Center........ & 004022.29 & $\begin{array}{lll}41 & 41 & 09.4\end{array}$ & 0.30 \\
\hline South & 004023.75 & 414010.3 & 0.55 \\
\hline Core .............................. & 004022.29 & 414109.4 & 0.07 \\
\hline 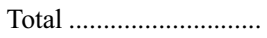 & 004022.38 & 414052.3 & 4.36 \\
\hline
\end{tabular}

NotE.-Units of right ascension are hours, minutes, and seconds, and units of declination are degrees, arcminutes, and arcseconds.

${ }^{a}$ Elliptical aperture, as shown in Fig. 4.

A comparison of the mid-/far-IR data with other tracers of the ISM, such as H I (Young \& Lo 1997), shows the same morphology (see Fig. 6), indicating that dust and gas are very well mixed in NGC 205. On the basis of the H I velocity data, it is clear that the $\mathrm{H}$ I gas rotates in the north-south direction with a velocity gradient of $42 \mathrm{~km} \mathrm{~s}^{-1}$ over 900 pc (Young \& Lo 1997). However, there is no clear one-to-one correspondence between the $\mathrm{H}$ I column density peaks and velocity, and therefore the three distinct IR emission regions are not necessarily dynamical entities. The observed dust morphology is similar at all wavelengths from 8 to $160 \mu \mathrm{m}$ (within the limits of varying angular resolution) and is also similar to the $\mathrm{H}$ I morphology seen in the $21 \mathrm{~cm}$ line. The data are consistent with an ISM in which the atomic gas and dust are well mixed, and therefore we see no evidence for significant stratification of the ISM in NGC 205.

\section{ADDITIONAL DATA PROCESSING}

A reliable estimate of the IR and diffuse emission in NGC 205 requires an estimate of the foreground point-source contamination arising either from the Milky Way (MW) or M31. Point sources were extracted in the central $5^{\prime} \times 5^{\prime}$ region of NGC 205

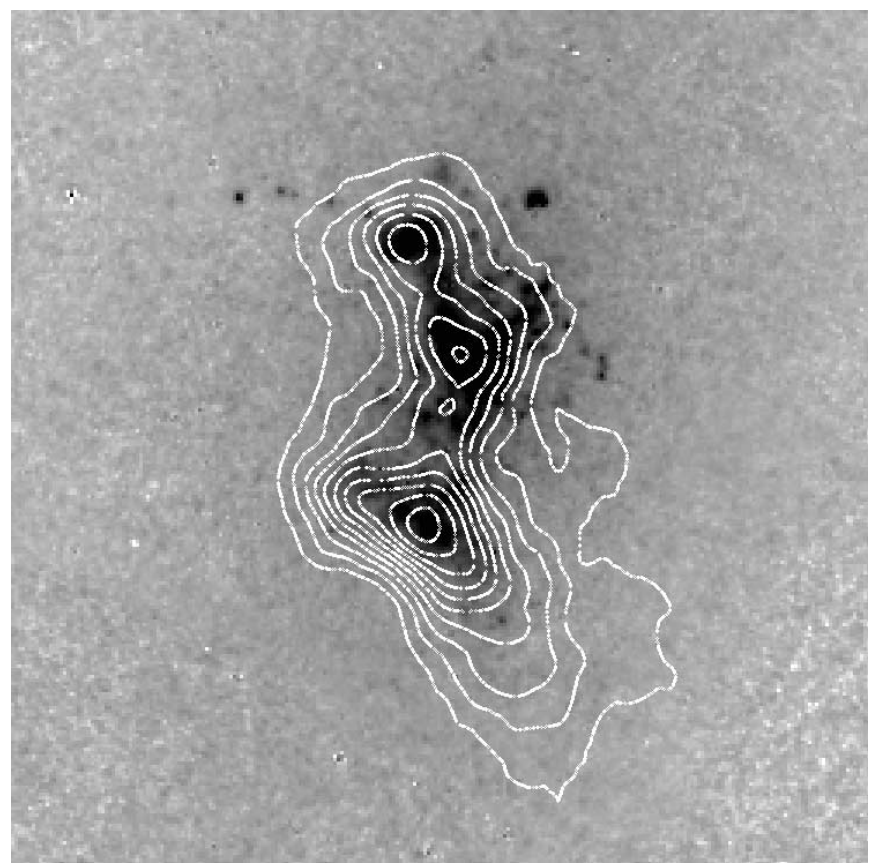

FIG. 6.-H I column density, derived by Young \& Lo (1997), overlaid on the IRAC $8 \mu \mathrm{m}$ gray-scale image of NGC 205 where point sources have been removed. The $\mathrm{H}$ I density contours range from $2 \times 10^{19}$ to $3.8 \times 10^{20} \mathrm{~cm}^{-2}$ in intervals of $4 \times 10^{19} \mathrm{~cm}^{-2}$. The FOV is $5^{\prime} \times 5^{\prime}$, with north up and east to the left. 

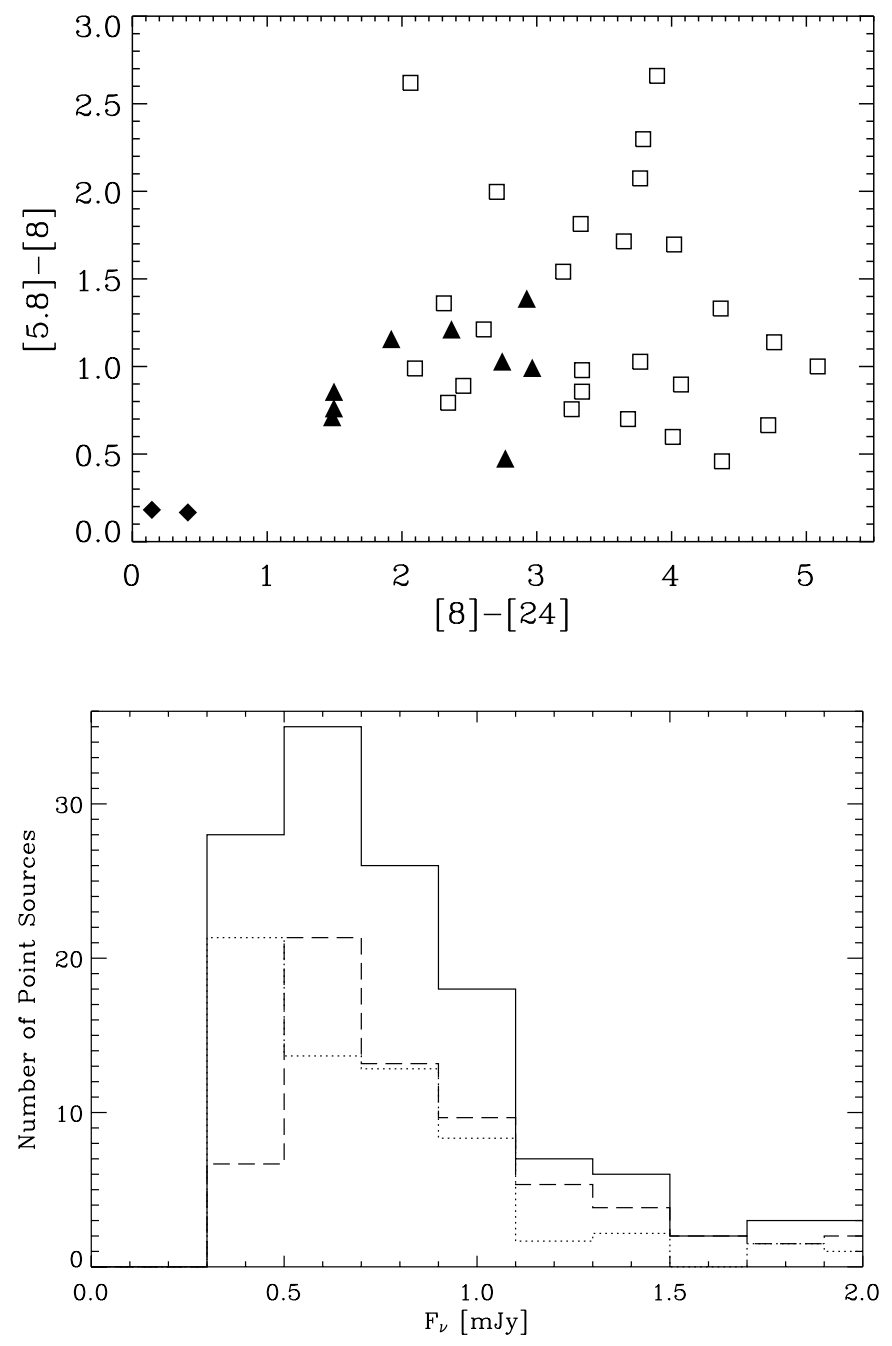

Fig. 7.-Top: Color-color diagram of a sample of K giant stars ( filled diamonds), AGB stellar templates ( filled triangles), and the point sources extracted in the central $5^{\prime}$ of the NGC 205 field (open squares). Bottom: Histogram showing the number of point sources as a function of $24 \mu \mathrm{m}$ flux in the central $5^{\prime}$ of the NGC 205 field (solid line), for the average of the six control fields (see Table 2; dashed line), and the remaining distribution for NGC 205 after the average from the control fields has been removed (dotted line).

in the 5.8, 8.0, and $24 \mu \mathrm{m}$ images using the source extraction software StarFinder (Diolaiti et al. 2000). Spitzer [8] - [24] and $[5.8]-[8]$ colors were derived for these point sources and were compared to the colors of a sample of Galactic AGB and K giant star templates in the mid-infrared observed with the Short Wavelength Spectrometer (SWS) on board ISO. Using ISAP (ISO Spectral Analysis Package, version 2.0), ${ }^{6}$ it is possible to determine the flux density within each Spitzer imager/photometer bandpass over the SWS spectra, which cover a wavelength range of 2.4$45.2 \mu \mathrm{m}$. The point sources in the central region of NGC 205 (Fig. 7, top, open squares) have colors similar to those of AGB stars ( filled triangles). However, $\mathrm{K}$ giant stars in the field are clearly identifiable due to their much "bluer" [8] - [24] color, and consequently we conclude that none are present in the central region of NGC 205.

The foreground contamination at $24 \mu \mathrm{m}$, from M31 point sources, where dusty AGBs contribute most of their flux, is estimated using six control fields. Each field has dimensions of $15^{\prime} \times 15^{\prime}$ to match that of NGC 205 and is located a comparable

\footnotetext{
${ }^{6}$ ISAP is available at http://www.ipac.caltech.edu/iso/isap/isap.html.
}

TABLE 2

NGC 205 Pointing and Control Fields

\begin{tabular}{|c|c|c|}
\hline Field & $\alpha(\mathrm{J} 2000.0)$ & $\delta(\mathrm{J} 2000.0)$ \\
\hline NGC $205 .$. & 004022.10 & 414107.1 \\
\hline 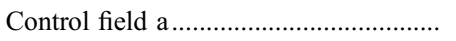 & 003638.65 & 403747.0 \\
\hline 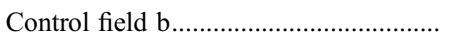 & 004454.11 & 405103.5 \\
\hline 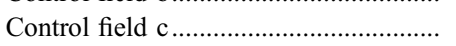 & 004336.11 & 403149.4 \\
\hline 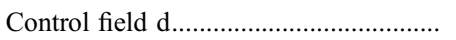 & 004624.97 & $41 \quad 1013.8$ \\
\hline 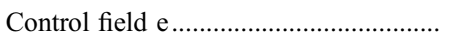 & 004738.73 & 412852.5 \\
\hline Control field f . & 004254.21 & 421951.6 \\
\hline
\end{tabular}

NoтE.-Units of right ascension are hours, minutes, and seconds, and units of declination are degrees, arcminutes, and arcseconds.

distance away from the center of M31 (Table 2). To determine the number of AGB stars in NGC 205, we count these stars in the central $15^{\prime} \times 15^{\prime}$ region and estimate the number of foreground stars by averaging their numbers in the six control fields. On the basis of the results shown in Figure 7 (bottom), we estimate that about half of the point sources in the NGC $20515^{\prime} \times 15^{\prime}$ field are from foreground contamination due either to the MW or M31.

We estimate that in the smaller Total region centered on NGC 205 (as defined in Table 1), about one-third of the point sources are from foreground contamination. Given a mean flux density of $0.6 \mathrm{mJy}$ for contaminating point sources (see Fig. 7, bottom), we estimate a flux density contamination of $\sim 2 \mathrm{mJy}$. This number is below our flux density measurement errors at $24 \mu \mathrm{m}$ (see Table 3), and therefore no further processing (i.e., point-source removal) is done to the $24 \mu \mathrm{m}$ image.

\subsection{Aperture Correction of Extended Sources}

We measure the IRAC 3.6, 4.5, 5.8, and $8 \mu \mathrm{m}$ and the MIPS 24,70 , and $160 \mu \mathrm{m}$ fluxes in the spatially resolved regions common to all wavelengths, as depicted in Figure 4 and listed in Table 1. The IRAC and MIPS data are combined with previous observations taken with $I S O$ at $14.3 \mu \mathrm{m}$ (Xilouris et al. 2004) and with the James Clerk Maxwell Telescope (JCMT) at $1.1 \mathrm{~mm}$ (Fich \& Hodge 1991). The flux uncertainty for each region includes the combined effect of the systematic errors due to the postprocessing of the images, plus that of the flux density calibration of the instruments (Fazio et al. 2004; Rieke et al. 2004). The flux uncertainty, therefore, was estimated by measuring the residual flux density away from the galaxy in the background-subtracted images and adding an absolute flux calibration uncertainty of $10 \%$ for the IRAC and MIPS $24 \mu \mathrm{m}$ measurements and of $20 \%$ for the ISO $14.3 \mu \mathrm{m}$ and MIPS 70 and $160 \mu \mathrm{m}$ fluxes. The $8 \mu \mathrm{m}$ fluxes are corrected (multiplied) by the effective aperture correction factor of $0.944(3.6 \mu \mathrm{m}), 0.937(4.5 \mu \mathrm{m}), 0.772(5.8 \mu \mathrm{m})$, and $0.737(8 \mu \mathrm{m})$ (Reach et al. 2005). The corrected flux densities and uncertainties are given in Table 3.

\subsection{Stellar Photospheric Component of Diffuse Emission}

The stellar photospheric component of the diffuse emission coming from NGC 205 itself must be removed before the analysis of the dust component. We conservatively assume that, in addition to the Two Micron All Sky Survey (2MASS) $J, H$, and $K$ bands, all the light in the 3.6 and 4.5 IRAC bands is photospheric in origin. The $J, H, K$, and IRAC 3.6 and $4.5 \mu \mathrm{m}$ filter response functions were multiplied with a PEGASE stellar model (Fioc \& Rocca-Volmerange 1997) with an age of $350 \mathrm{Myr}$ (see Fig. 9) and integrated over wavelength, yielding fluxes that can be compared with the observed fluxes. The age was picked to roughly coincide with the (uncertain) epoch of recent star formation in 
TABLE 3

Photometry of Emission Regions in NGC 205

\begin{tabular}{|c|c|c|c|c|c|}
\hline \multirow{2}{*}{$\begin{array}{c}\text { WAVELENGTH } \\
(\mu \mathrm{m})\end{array}$} & \multicolumn{5}{|c|}{ Flux Density (mJy) } \\
\hline & North & Center & South & Core & Total \\
\hline $1.22 \ldots \ldots \ldots \ldots \ldots \ldots$ & $430 \pm 2$ & $130 \pm 7$ & $110 \pm 6$ & $46.2 \pm 2$ & $940 \pm 47$ \\
\hline $1.65 \ldots \ldots \ldots \ldots \ldots \ldots$ & $550 \pm 3$ & $160 \pm 8$ & $40 \pm 7$ & $56.8 \pm 3$ & $116 \pm 58$ \\
\hline 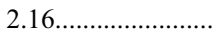 & $380 \pm 2$ & $110 \pm 6$ & $100 \pm 5$ & $39.1 \pm 2$ & $820 \pm 41$ \\
\hline 3.6............................... & $21 \pm 3$ & $59 \pm 6$ & $54 \pm 7$ & $23.5 \pm 2$ & $438 \pm 52$ \\
\hline $4.5 \ldots \ldots \ldots \ldots \ldots \ldots$ & $13 \pm 2$ & $35 \pm 4$ & $33 \pm 5$ & $14.9 \pm 1$ & $266 \pm 33$ \\
\hline $5.8 \ldots \ldots \ldots \ldots \ldots \ldots \ldots$ & $15 \pm 3$ & $33 \pm 5$ & $39 \pm 9$ & $14.2 \pm 1$ & $279 \pm 62$ \\
\hline 8 & $12 \pm 1$ & $26 \pm 3$ & $26 \pm 3$ & $14.5 \pm 1$ & $148 \pm 15$ \\
\hline 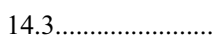 & $7 \pm 2$ & $15 \pm 3$ & $25 \pm 5$ & $5.9 \pm 1$ & $101 \pm 23$ \\
\hline $24 \ldots \ldots \ldots \ldots \ldots \ldots$ & $10 \pm 4$ & $18 \pm 5$ & $26 \pm 10$ & $5.8 \pm 1$ & $141 \pm 23$ \\
\hline $70 \ldots \ldots \ldots \ldots \ldots$ & $104 \pm 21$ & $181 \pm 36$ & $301 \pm 60$ & $61.7 \pm 12$ & $1428 \pm 290$ \\
\hline $160 \ldots \ldots \ldots \ldots \ldots \ldots \ldots$ & $473 \pm 279$ & $713 \pm 363$ & $1490 \pm 692$ & $253 \pm 45$ & $8761 \pm 4720$ \\
\hline
\end{tabular}

NGC 205 (see, e.g., Butler \& Martinez-Delgado 2005; Davidge 2005). There is no diagnostic power in the age that we assumed; we used synthetic spectra from simple stellar population (SSP) models only to estimate the stellar contribution to the Spitzer bands, as simple power laws cannot reproduce the $J, H$, and $K$ colors. Scalings were derived for each band individually and were averaged together to get the final scaling for the photospheric component. In all cases, the fluxes from $J$ to $4.5 \mu \mathrm{m}$ are consistent with being completely stellar in origin. To estimate the photospheric contribution to the flux density at longer wavelengths, we multiplied and integrated over wavelength the relevant IRAC and MIPS bandpasses with the PEGASE model and subtracted the result from the observed flux density to yield an estimate for the total dust flux density. For the relative contributions of the diffuse photospheric emission to the observed fluxes, see Table 4. The age we use for the PEGASE models (within a factor of 3) does not significantly alter the dust fits; indeed, PEGASE models using ages between 0.1 and 1 Gyr result in very similar estimates for the stellar contamination in the Spitzer bands, especially for $\lambda \geq 8 \mu \mathrm{m}$.

\section{MASS OF DUST AND GAS IN NGC 205}

With these new Spitzer observations, it is possible to derive the mass of dust and estimate the gas mass in NGC 205 using different methods and to compare the results, both for the total emission and within the three main emission regions. In the first method, we use a single-temperature fit to the 70 and $160 \mu \mathrm{m}$ data to determine the dust temperature, assuming a modified blackbody emissivity function of the form

$$
F(\lambda)=\lambda^{-\beta} \frac{2 h c^{2} \lambda^{-5}}{e^{h c / k \lambda T}-1} .
$$

We adopt an emissivity coefficient $\beta=2$ (valid beyond $70 \mu \mathrm{m}$; cf. Draine 2003). If we assume a gas-to-dust mass ratio of $\sim 100$, representative of the solar neighborhood and the inner disk of the Milky Way (Hildebrand 1983; Devereux \& Young 1990), the dust temperature and emissivity can be used to estimate the mass of the dust and gas. This method has been extensively used to determine dust masses in galaxies, but it has a few drawbacks (Devereux \& Young 1990; Dale \& Helou 2002) and should be taken as an approximation. A fit to the spectral energy distribution (SED) has been shown to provide better estimates to the dust masses for each dust grain component (see below).

A reliable ratio of the far-IR fluxes is a necessary ingredient to get the best temperature estimate. The angular resolution of the
TABLE 4

Stellar Photospheric and Dust Flux Density Contributions

\begin{tabular}{|c|c|c|c|c|c|}
\hline Region & $\begin{array}{c}\text { Wavelength } \\
(\mu \mathrm{m})\end{array}$ & $\begin{array}{l}\text { Total }^{\mathrm{a}} \\
(\mathrm{mJy})\end{array}$ & $\begin{array}{l}\text { Dust } \\
\text { (mJy) }\end{array}$ & Stellar ${ }^{\mathrm{b}}$ & Dust Fraction \\
\hline \multirow[t]{8}{*}{ North .................. } & 3.6 & 20.8 & 0.0 & 1.0 & 0.00 \\
\hline & 4.5 & 12.6 & 0.0 & 0.60 & 0.00 \\
\hline & 5.8 & 15.2 & 6.4 & 0.42 & 0.42 \\
\hline & 8.0 & 11.7 & 6.6 & 0.25 & 0.56 \\
\hline & 14.3 & 6.9 & 5.2 & 0.08 & 0.75 \\
\hline & 24.0 & 10.1 & 9.5 & 0.03 & 0.94 \\
\hline & 70.0 & 104.2 & 104.2 & 0.00 & 1.00 \\
\hline & 160.0 & 472.9 & 472.9 & 0.00 & 1.00 \\
\hline \multirow{8}{*}{ Center............... } & 3.6 & 58.6 & 0.0 & 1.0 & 0.00 \\
\hline & 4.5 & 35.4 & 0.0 & 0.60 & 0.00 \\
\hline & 5.8 & 32.6 & 7.2 & 0.43 & 0.22 \\
\hline & 8.0 & 25.5 & 10.7 & 0.25 & 0.42 \\
\hline & 14.3 & 14.9 & 9.8 & 0.08 & 0.66 \\
\hline & 24.0 & 17.6 & 15.7 & 0.03 & 0.89 \\
\hline & 70.0 & 181.5 & 181.5 & 0.00 & 1.00 \\
\hline & 160.0 & 712.9 & 712.9 & 0.00 & 1.00 \\
\hline \multirow[t]{8}{*}{ South ................. } & 3.6 & 54.1 & 0.0 & 1.0 & 0.00 \\
\hline & 4.5 & 32.8 & 0.0 & 0.60 & 0.00 \\
\hline & 5.8 & 38.8 & 15.5 & 0.40 & 0.40 \\
\hline & 8.0 & 25.7 & 12.1 & 0.23 & 0.47 \\
\hline & 14.3 & 25.5 & 20.9 & 0.09 & 0.82 \\
\hline & 24.0 & 25.8 & 24.0 & 0.03 & 0.93 \\
\hline & 70.0 & 300.8 & 300.8 & 0.00 & 1.00 \\
\hline & 160.0 & 1490.0 & 1490.0 & 0.00 & 1.00 \\
\hline \multirow[t]{8}{*}{ Total .................. } & 3.6 & 437.6 & 0.0 & 1.0 & 0.00 \\
\hline & 4.5 & 265.7 & 0.0 & 0.61 & 0.00 \\
\hline & 5.8 & 279.0 & 89.3 & 0.43 & 0.32 \\
\hline & 8.0 & 147.9 & 37.0 & 0.25 & 0.25 \\
\hline & 14.3 & 101.4 & 63.9 & 0.09 & 0.63 \\
\hline & 24.0 & 141.4 & 127.2 & 0.03 & 0.90 \\
\hline & 70.0 & 1428.0 & 1428.0 & 0.00 & 1.00 \\
\hline & 160.0 & 8761.3 & 8761.3 & 0.00 & 1.00 \\
\hline \multirow[t]{9}{*}{ Core.................... } & 3.6 & 23.1 & 0.0 & 1.0 & 0.00 \\
\hline & 4.5 & 14.9 & 0.0 & 0.64 & 0.00 \\
\hline & 5.8 & 14.2 & 5.1 & 0.40 & 0.36 \\
\hline & 8.0 & 14.5 & 9.3 & 0.22 & 0.64 \\
\hline & 14.3 & 5.9 & 4.1 & 0.08 & 0.69 \\
\hline & 24.0 & 5.1 & 4.4 & 0.03 & 0.87 \\
\hline & 70.0 & 61.7 & 61.7 & 0.00 & 1.00 \\
\hline & 160.0 & 253.2 & 253.2 & 0.00 & 1.00 \\
\hline & 1100.0 & 21.0 & 21.0 & 0.00 & 1.00 \\
\hline
\end{tabular}

a Measured flux density (photospheric plus dust contributions).

b Stellar flux density normalized with respect to that at $3.6 \mu \mathrm{m}$. 


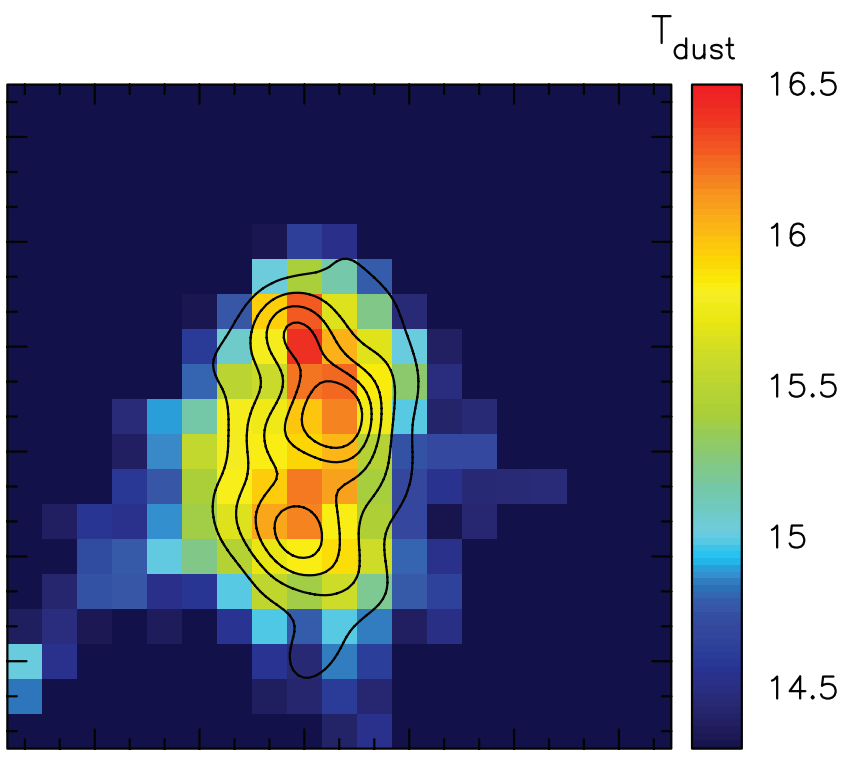

FIG. 8.-Dust temperature of NGC 205 within the central $5^{\prime}$, with $24 \mu \mathrm{m}$ contours shown for levels of $0.3,0.35,0.4,0.45$, and $0.5 \mathrm{MJy} \mathrm{sr}^{-1}$. The temperatures are based on the 70 and $160 \mu \mathrm{m}$ flux ratio. The 24 and $70 \mu \mathrm{m}$ images have been convolved to the $160 \mu \mathrm{m}$ resolution.

$70 \mu \mathrm{m}$ image was reduced to match that at $160 \mu \mathrm{m}$ by convolving the image with the $160 \mu \mathrm{m}$ kernel, derived from the pointspread function model generated using the Tiny Tim software (Krist \& Hook 2002). The IRAF routines geomap and geotran were then used to match the pixel scale and astrometry, preserving the flux in the transformation. Each pixel is assigned a temperature on the basis of the best fit to the modified blackbody function. The resulting temperature map is shown in Figure 8. The temperature is very uniform, ranging from $\sim 14$ to $17 \mathrm{~K}$ in the innermost $5^{\prime}$ that encompasses the three main emission regions. In the central rectangular region of size 2 ! $1 \times 2.9$ (or an area of $6.3 \operatorname{arcmin}^{2}$, slightly larger than the Total region as defined in Table 1), we calculate the dust mass from the $160 \mu \mathrm{m}$ emission using the following equation:

$$
M_{d}=S_{\nu} D^{2} / \kappa_{d} B_{\nu, T}
$$

where $D$ is the distance to the galaxy $(815 \mathrm{kpc})$ and $\kappa_{d}$ is the mass absorption coefficient, for which we assume a value of $6.73 \mathrm{~m}^{2} \mathrm{~kg}^{-1}$ at $70 \mu \mathrm{m}$ and $1.2 \mathrm{~m}^{2} \mathrm{~kg}^{-1}$ at $160 \mu \mathrm{m}$ (Li \& Draine 2001). The total dust mass in this region, derived from the sum of the individual mass estimates for each pixel, is $(6.1 \pm 1.2) \times$ $10^{4} M_{\odot}$, yielding a gas mass of $6.1 \times 10^{6} M_{\odot}$ for a gas-to-dust mass ratio of 100 .

Alternatively, we can determine dust masses by fitting the total dust emission SED. The SED was measured between 5.8 and $160 \mu \mathrm{m}$, using IRAC, ISO $14.3 \mu \mathrm{m}$, and MIPS data. We measure the fluxes in the spatially resolved regions common to all wavelengths, as depicted in Figure 4 and listed in Table 3.

The dust emission in NGC 205 was modeled by an equation of the form

$$
F_{\text {dust }}(\lambda)=\sum C_{i} \kappa_{i}(\lambda) B_{\lambda}\left(T_{D, i}\right)
$$

where the sum extends over $i$ dust components, $C_{i}=M_{d, i} / D^{2}$, $D$ is as in equation (2), and $\kappa_{i}$ is the mass absorption coefficient of the $i$ th dust component. For NGC 205, the relatively large 5.8, 8 , and $14.3 \mu \mathrm{m}$ fluxes require a hot component, which we ascribe to PAH molecules. While the 70 and $160 \mu \mathrm{m}$ emission could be fitted with a single temperature component, including the $24 \mu \mathrm{m}$ and $1.1 \mathrm{~mm}$ fluxes requires additional temperature components. To satisfactorily fit the full SED of NGC 205, we adopt a threecomponent model: warm and cold silicates $(a \sim 0.1 \mu \mathrm{m})$ and PAHs, the former to reproduce the $24-1100 \mu \mathrm{m}$ emission and the latter to reproduce the 5.8-24 $\mu \mathrm{m}$ emission. Mass absorption coefficients for astronomical silicates were computed from Mie theory using the dielectric functions of Laor \& Draine (1993). Cross sections for the PAH molecules were taken from $\mathrm{Li} \&$ Draine (2001). As the canonical PAH spectrum (e.g., NGC 7027; Werner et al. 2004) exhibits no features beyond $20 \mu \mathrm{m}$, we recomputed the PAH cross sections (and mass absorption coefficients), leaving off the last three terms of Li \& Draine's equation (11). The PAH component is included here for completeness of the fit, but we do not use the temperature or mass estimates, as the PAH emission is a stochastic process rather than an equilibrium process, as assumed in equation (3).

With only six measurements and six parameters $\left(C_{1,2,3}, T_{D_{1,2,3}}\right)$, a formal fit is not fully constrained, and we proceed by making a detailed search of the parameter space. With our assumptions about the components present in the observed SED, we searched a limited set of parameters for dust mass (through $C_{i}$ ) and temperature for each component. We assumed temperature ranges of $200-550 \mathrm{~K}, 25-150 \mathrm{~K}$, and 8-25 K for the PAHs, warm silicates, and cold silicates, respectively. At each set of temperature points, a similar grid of mass scalings $\left(C_{i}\right)$ was explored for each component. After an initial, coarse grid search, a finer temperature and mass scaling grid was defined for each component and the procedure was repeated. We iterate this procedure (typically only two refinements of the grid were needed) until a good fit in the $\chi^{2}$ sense was found. We estimated uncertainties in the derived quantities by examining the distribution of $\chi^{2}$ about the minimum for all input parameter sets (typically $1 \times 10^{5}-1 \times 10^{6}$ points in the six-dimensional parameter space). While the results of this procedure are not unique, it yields representative estimates of both the dust mass and temperature, given the input assumptions. In all cases the dust mass is dominated by the cold component and should be considered as a weak lower limit. Due to the lack of data beyond $160 \mu \mathrm{m}$, we cannot formally rule out much larger dust masses. However, several pieces of evidence argue against dust masses at the upper end of the formal error bars. First, where we do have submillimeter data (the Core region), the dust mass is consistent with much lower upper "limits" on the mass, and second, the upper error limits would imply very large fluxes at submillimeter wavelengths, which is not what is observed (Fich \& Hodge 1991).

TABLE 5

\begin{tabular}{|c|c|c|c|c|}
\hline \multirow[b]{2}{*}{ REGION } & \multicolumn{2}{|c|}{ Warm Silicates } & \multicolumn{2}{|c|}{ Cold Silicates } \\
\hline & $\begin{array}{l}\text { Mass } \\
\left(M_{\odot}\right)\end{array}$ & $\begin{array}{c}\text { Temperature } \\
(\mathrm{K})\end{array}$ & $\begin{array}{l}\text { Mass } \\
\left(M_{\odot}\right)\end{array}$ & $\begin{array}{c}\text { Temperature } \\
\text { (K) }\end{array}$ \\
\hline North ......... & $1.2_{-1}^{+3}$ & $53_{-5}^{+8}$ & $2.2_{-1.5}^{+29} \times 10^{3}$ & $17.5_{-7}^{+5}$ \\
\hline Center........ & $2.1_{-2}^{+4}$ & $53_{-6}^{+11}$ & $2.9^{+28} \times 10^{3}$ & $17_{-8}^{+3}$ \\
\hline South ......... & $5.3_{-5}^{+4}$ & $51_{-3}^{+18}$ & $1.2_{-0.9}^{+2.7} \times 10^{4}$ & $15.8_{-3}^{+5}$ \\
\hline Core $^{\mathrm{a}} \ldots \ldots \ldots$ & $0.6_{-0.3}^{+2.5}$ & $53_{-8}^{+3}$ & $1.1_{-09}^{+30} \times 10^{3}$ & $17.8_{-10}^{+5}$ \\
\hline Core $^{\mathrm{b}}$. & $1.9_{-1}^{+2.3}$ & $48_{-4}^{+3}$ & $1.8_{-0.4}^{+1.1} \times 10^{4}$ & $11.6_{-2}^{+3}$ \\
\hline Total .......... & $6.1_{-5}^{+13}$ & $58_{-5}^{+25}$ & $3.2_{-1.8}^{+1.4} \times 10^{4}$ & $18.1_{-2}^{+2}$ \\
\hline
\end{tabular}

Mass and Temperature Estimates from SED Fits

a SED without the $1.1 \mathrm{~mm}$ flux density measurement.

b SED with the $1.1 \mathrm{~mm}$ flux density measurement. 

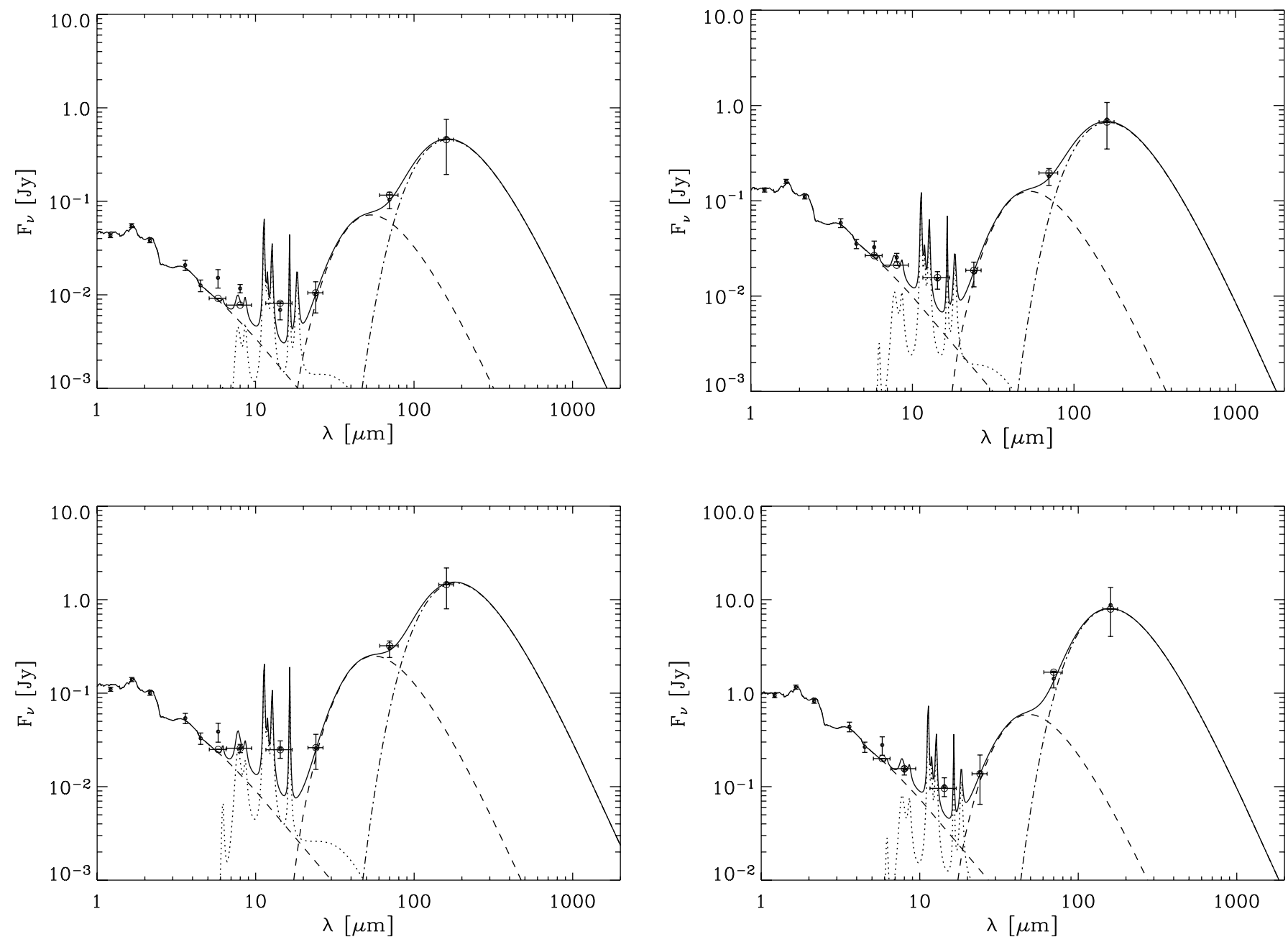

FIG. 9.- Infrared SEDs of the North (top left), Center (top right), South (bottom left), and Total (bottom right) regions of NGC 205 (see Table 1). The diagrams

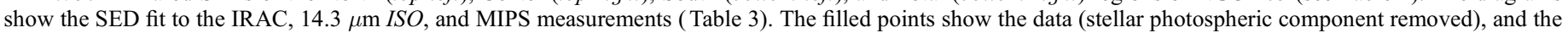
open points show the model multiplied with the passband and integrated over wavelength. The dotted line indicates the PAHs component, the dashed line indicates the warm silicates component, the dot-dashed line indicates the cold silicates component, and the solid line indicates the total of all the components.

The best SED fits to the data for each region, which produce the dust masses and temperatures given in Table 5, are shown in Figures 9 and 10. Using the same gas-to-dust mass ratio of 100 , we infer a gas mass for the Total region of $3.2 \times 10^{6} M_{\odot}$. This value is nearly one-half of the $6.1 \times 10^{6} M_{\odot}$ mass derived from the 70/160 flux ratio method. Summing up the total dust mass in the three distinct large regions using the best-fit values, we estimate a gas mass of $1.7 \times 10^{6} M_{\odot}$; that is, smaller than that of the Total region. This result suggests that a larger reservoir of gas/ dust with a comparable gas mass surrounds the three regions, as first suggested by the H I (Young \& Lo 1997) and CO (Welch et al. 1998) observations.

The Core region has a detection at $1.1 \mathrm{~mm}$ (Fich \& Hodge 1991), and adding this value to its SED and computing a new fit results in a dust mass of $1.8 \times 10^{4} M_{\odot}$; that is, approximately a factor of 16 larger than the mass estimated without the $1.1 \mathrm{~mm}$ point $\left(1.1 \times 10^{3} M_{\odot}\right)$, and at a colder temperature, $11.6 \mathrm{~K}(\mathrm{com}$ pared to $17.8 \mathrm{~K}$ ). One can speculate that if this colder dust component has a similar distribution across our selected regions, preserving the same mass gas-to-dust ratio, then one predicts a gas mass several times larger, $\sim 5 \times 10^{7} M_{\odot}$. This mass determination is consistent with the fact that adding submillimeter measurements to far-IR observations of early-type galaxies usually yields larger cold dust mass estimates (as much as a factor of 10)

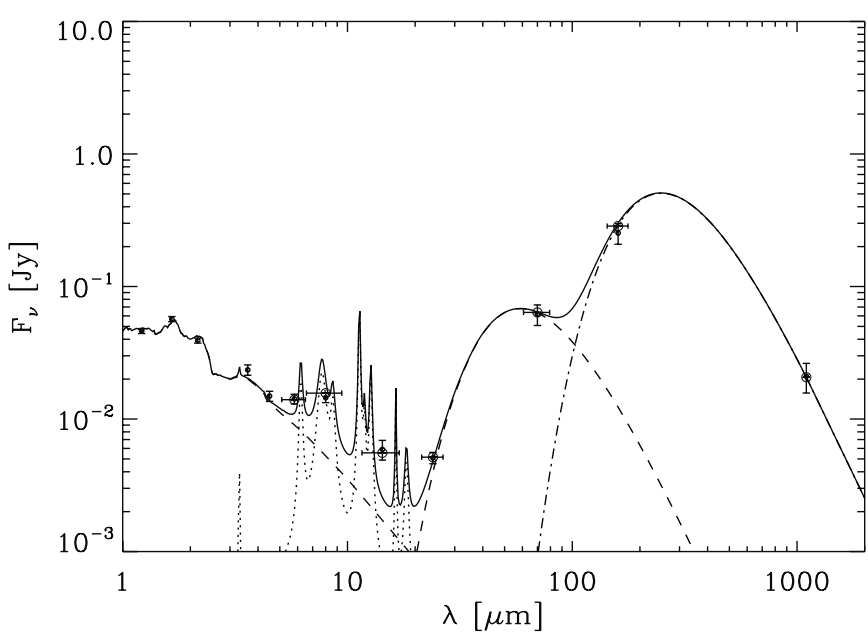

FIG. 10.- Infrared SEDs of the Core region of NGC 205, as defined in Table 1. The diagram shows the SED fit to the IRAC, $14.3 \mu \mathrm{m} I S O$, and MIPS measurements ( Table 3), plus the $1.1 \mathrm{~mm}$ data point measured by Fich \& Hodge (1991). The symbols and line styles are defined in Fig. 9. 
than the far-IR measurements alone (Devereux \& Young 1990; Temi et al. 2004).

\section{DISCUSSION}

One of the open issues in understanding the ISM of NGC 205 has been its "missing ISM mass"; that is, the apparent lack of gas in comparison with the expected mass return to the interstellar medium by evolved stars (Welch et al. 1998). By determining the dust mass and selecting a reasonable gas-to-dust mass ratio (e.g., 100), one can estimate the amount of gas in a straightforward manner.

The dust mass estimate for the Core region of NGC 205 from Fich \& Hodge (1991), using IRAS data and including $1.1 \mathrm{~mm}$ observations, is $M_{\text {dust }}=1-3 \times 10^{3} M_{\odot}$, depending on the adopted value for the dust emissivity. The recent mass dust determination in the same region by Haas (1998), adding ISO data between 120 and $200 \mu \mathrm{m}$ to the SED, is $M_{\text {dust }}=0.88 \times 10^{3} M_{\odot}$ without the $1.1 \mathrm{~mm}$ data and $M_{\text {dust }}=1.18 \times 10^{4} M_{\odot}$ including it (for a emissivity exponent $\beta=2$ ); that is, a factor of 4 higher than Fich \& Hodge's upper limit. Using the Spitzer data alone, we estimate for the Core region a value of $M_{\text {dust }}=1.1 \times 10^{3} M_{\odot}$. If we include the $1.1 \mathrm{~mm}$ data, the mass estimate increases to $M_{\text {dust }}=$ $1.8 \times 10^{4} M_{\odot}$. This latter value is similar to that obtained by Haas, except that our dust temperature is $11.6 \mathrm{~K}$ rather than $8 \mathrm{~K}$. Therefore, the mass of gas, for a gas-to-dust ratio of 100, for the NGC 205 Core region is $M_{\text {gas }}=1.8 \times 10^{6} M_{\odot}$.

Using only our far-IR Spitzer data for the SED, we determine a Total amount of dust at $18 \mathrm{~K}$ of $M_{\text {dust }}=3.2 \times 10^{4} M_{\odot}$, which implies a mass of $M_{\text {gas }}=3.2 \times 10^{6} M_{\odot}$ for the gas. This latter value is 4 times larger than the ISM mass determination of $M_{\mathrm{ISM}}=7.2 \times 10^{5} M_{\odot}$ by Sage et al. (1998) and is in perfect agreement with the theoretical gas mass estimate of $M_{\text {gas }}=2.6 \times$ $10^{6} M_{\odot}$ generated by the injection of gas by evolved stars over 0.5 Gyr. This comparison suggests that the mass determination by Sage et al. (1998) is indeed a lower limit to the ISM mass, and that essentially over our Total region one can account for all the ISM expected in NGC 205 from the highly evolved stars since the last starburst.

If the mass of dust in NGC 205 is indeed a few times larger than previous estimates, then we believe this provides a more coherent picture of its recent star formation history. The global evolutionary path of the ISM in NGC 205 was summarized by Welch et al. (1998). They pointed out that any reasonable efficiency during the last starburst in NGC 205 implies a more massive ISM. Their idea is straightforward: if the burst transformed $\sim 1.4 \times 10^{6} M_{\odot}$ of gas into stars (the Wilcots et al. 1990 value, adjusted to include low-mass stars $<1 M_{\odot}$ ) with a $10 \%$ efficiency, then NGC 205 must have had at least $\sim 1.2 \times 10^{7} M_{\odot}$ of gas. Our dust mass estimates could easily account for at least $\sim \frac{1}{3}$ of such a gas reservoir, and nearly all of the gas temperatures were $\sim 14 \mathrm{~K}$. Finally, a burst of $\sim 1.4 \times 10^{6} M_{\odot}$ would yield $\sim 90$ stars in the brightest magnitude of the AGB according to the fuel-burning theorem of Renzini \& Buzzoni (1986; assuming a mass-to-light ratio of 0.2 , the same as that of NGC 1866, a $10^{8} \mathrm{yr}$ old star cluster; Fischer et al. 1992). This is of the same order of magnitude as what we see in Figure 7 (bottom).

\section{SUMMARY}

New IRAC and MIPS observations of NGC 205 give a better assessment of the total dust mass in this prototypical LDG system. The Spitzer data have been complemented with ISOCAM archival data to create a well-defined spectral energy distribution in the 3.6-160 $\mu \mathrm{m}$ wavelength range. The higher sensitivity and angular resolution of IRAC and MIPS provide a detailed view of the morphology of the interstellar medium in NGC 205. At 8 and $24 \mu \mathrm{m}$ the spatial structure of the warm dust is clearly resolved. Although the 8 and $24 \mu \mathrm{m}$ images resemble the drawings of the dust clouds inferred by Hodge (1973) using isodensity measurements of the ground-based visual photographic plates (cf. Welch et al. 1998, Fig. 1; Young 2000, Fig. 1), these new IR data display a more complex structure. To facilitate our analysis of the color temperature and dust masses, we identified three main regions. As shown in Figure 2, the dust clouds within these regions are bright and conspicuous at the longer wavelength, too, having sizes ranging from $\sim 100$ to $300 \mathrm{pc}$ and dust masses of $\sim 10^{3}-10^{4} M_{\odot}$. A larger Total area encompasses the three regions and covers most of the bright $160 \mu \mathrm{m}$ dust emission.

The derived dust masses, based on the 70/160 $\mu \mathrm{m}$ flux ratio and a best fit to the SED, imply gas masses (assuming a standard gas-to-dust mass ratio of 100; cf. Young 2000) that are consistent with each other and are larger $\left(3.2-6.1 \times 10^{6} M_{\odot}\right)$ than previously estimated from $\mathrm{CO}$ and $\mathrm{H}$ I observations. This indicates that a significant contribution to the dust and gas masses is coming from a fainter component surrounding the brightest emission regions. Adding the $1.1 \mathrm{~mm}$ detection to our measurements of the Core region of NGC 205 increases the estimate of the cold dust in that region by a factor of 16 . Our gas mass estimate in NGC 205 is a factor of 4 larger than previously detected, but it could be still larger if the distribution of the very cold dust $(\sim 12-14 \mathrm{~K})$ spreads out to the three selected regions. If this is the case, the gas mass would be 16 times that of the Total region (Table 5); that is, $5 \times 10^{7} M_{\odot}$ for a gas-to-dust mass ratio of 100 . Overall, the gas estimates are therefore consistent with the predicted mass return from dying stars, based on the last burst of star formation, $5 \times 10^{8} \mathrm{yr}$ ago.

This work is based on observations made with the Spitzer Space Telescope, which is operated by the Jet Propulsion Laboratory (JPL), California Institute of Technology, under NASA contract 1407 . Support for this work was provided by NASA and through JPL contract 1255094. We thank the referee for her/his careful reading of the manuscript.
Baade, W. 1944, ApJ, 100, 137

1951, Publ. Obs. Univ. Michigan, 10, 7

Bertola, F., Bressan, A., Burstien, D., Buson, L. M., Chiosi, C., \& di Serego Alighieri, S. 1995, ApJ, 438, 680

Blommaert, J., et al. 2003, The ISO Handbook, Volume II: CAM-The ISO

Camera, ver. 2.0 (ESA SP-1262; Noordwijk: ESA)

Butler, D. J., \& Martinez-Delgado, D. 2005, AJ, 129, 2217

Choi, P. I., Guhathakurta, P., \& Johnston, K. 2002, AJ, 124, 310

Coulais, A., \& Abergel, A. 2000, A\&AS, 141, 533

Dale, D., \& Helou, G. 2002, ApJ, 576, 159

Davidge, T. J. 2003, ApJ, 597, 289

. 2005, AJ, 130, 2087

\section{REFERENCES}

Désert, F.-X., Boulanger, F., \& Puget, J. L. 1990, A\&A, 237, 215

Devereux, N. A., \& Young, J. S. 1990, ApJ, 359, 42

Diolaiti, E., Bendinelli, O., Bonaccini, D., Close, L., Currie, D., \& Parmeggiani, G. 2000, A\&AS, 147, 335

Draine, B. T. 2003, ARA\&A, 41, 241

Draine, B. T., \& Li, A. 2001, ApJ, 551, 807

Faber, S. M., \& Gallagher, J. S. 1976, ApJ, 204, 365

Fazio, G. G., et al. 2004, ApJS, 154, 10

Ferguson, H. C., \& Binggeli, B. 1994, A\&A Rev., 6, 67

Ferrari, F., Pastoriza, M. G., Macchetto, F. D., Bonatto, C., Panagia, N., \& Sparks, W. B. 2002, A\&A, 389, 355

Fich, M., \& Hodge, P. W. 1991, ApJ, 374, L17 
Fioc, M., \& Rocca-Volmerange, B. 1997, A\&A, 326, 950

Fischer, P., Welch, D. L., Côté, P., Mateo, M., \& Madore, B. F. 1992, AJ, 103, 857

Gordon, K. D., et al. 2006, ApJ, 638, L87

Grebel, E. K. 2005, in AIP Conf. Proc. 752, Stellar Astrophysics with the World's Largest Telescopes, ed. J. Mikolajewska \& A. Olech (New York: AIP), 161

Haas, M. 1998, A\&A, 337, L1

Hildebrand, R. H. 1983, QJRAS, 24, 267

Hodge, P. W. 1973, ApJ, 182, 671 1989, ARA\&A, 27, 139

Ibata, R., Irwin, M., Lewis, G., Ferguson, A. M. N., \& Tanvir, N. 2001, Nature, 412, 49

Jura, M., Kim, D. W., Knapp, G. R., \& Guhathakurta, P. 1987, ApJ, 312, L11

Knapp, G. R., Guhathakurta, P., Kim, D.-W., \& Jura, M. A. 1989, ApJS, 70, 329

Krist, J., \& Hook, R. 2002, The Tiny Tim User's Guide, Version 6.0 (Baltimore: STScI)

Laor, A., \& Draine, B. T. 1993, ApJ, 402, 441

Li, A., \& Draine, B. T. 2001, ApJ, 554, 778 . 2002, ApJ, 572, 232

Mateo, M. 1998, ARA\&A, 36, 435

McConnachie, A. W., Irwin, M. J., Ferguson, A. M. N., Ibata, R. A., Lewis, G. F., \& Tanvir, N. R. 2005, MNRAS, 356, 979

McConnachie, A. W., Irwin, M. J., Lewis, G. F., Ibata, R. A., Chapman, S. C., Ferguson, A. M. N., \& Tanvir, N. R. 2004, MNRAS, 351, L94
Mould, J., Kristian, J., \& Da Costa, G. S. 1984, ApJ, 278, 575

Nilson, P. 1973, Uppsala General Catalogue of Galaxies (Uppsala: Uppsala Astron. Obs.)

Reach, W., et al. 2005, PASP, 117, 978

Renzini, A., \& Buzzoni, A. 1986, in Spectral Evolution of Galaxies, ed. C. Chiosi \& A. Renzini (Dordrecht: Reidel), 195

Rice, W., Lonsdale, C. J., Soifer, B. T., Neugebauer, G., Kopan, E. L., Lloyd, L. A., de Jong, T., \& Habing, H. J. 1988, ApJS, 68, 91

Rich, R. M., Corsi, C. E., Cacciari, C., Federici, L., Fusi Pecci, F., Djorgovski, S. G., \& Freedman, W. L. 2005, AJ, 129, 2670

Rieke, G. H., et al. 2004, ApJS, 154, 25

Sage, L. J., Welch, G. A., \& Mitchell, G. F. 1998, ApJ, 507, 726

Temi, P., Brighenti, F., Mathews, W. G., \& Bregman, J. D. 2004, ApJS, 151, 237

Welch, G. A., Sage, L. J., \& Mitchell, G. F. 1998, ApJ, 499, 209

Werner, M., et al. 2004, ApJS, 154, 309

Wiklind, T., \& Henkel, C. 1995, A\&A, 297, L71

Wilcots, E. M., Hodge, P., Eskridge, P. B., Bertola, F., \& Buson, L. 1990, ApJ, 364,87

Xilouris, E. M., Madden, S. C., Galliano, F., Vigroux, L., \& Sauvage, M. 2004, A\&A, 416, 41

Young, L. M. 2000, AJ, 120, 2460

Young, L. M., \& Lo, K. Y. 1997, ApJ, 476, 127 